\title{
New mass bound on fermionic dark matter from a combined analysis of classical dSphs
}

\author{
D. Savchenko ${ }^{1 \star}$ and A. Rudakovskyi ${ }^{1}$ \\ ${ }^{1}$ Bogolyubov Institute of Theoretical Physics, Metrolohichna Str. 14-b, 03143, Kyiv, Ukraine
}

Accepted XXX. Received YYY; in original form ZZZ

\begin{abstract}
Dwarf spheroidal galaxies (dSphs) are the most compact dark matter-dominated objects observed so far. The Pauli exclusion principle limits the number of fermionic dark matter particles that can compose a dSph halo. This results in a well-known lower bound on their particle mass. So far, such bounds were obtained from the analysis of individual dSphs. In this paper, we model dark matter halo density profiles via the semi-analytical approach and analyse the data from eight 'classical' dSphs assuming the same mass of dark matter fermion in each object. First, we find out that modelling of Carina dSph results in a much worse fitting quality compared to the other seven objects. From the combined analysis of the kinematic data of the remaining seven 'classical' dSphs, we obtain a new $2 \sigma$ lower bound of $m \gtrsim 190 \mathrm{eV}$ on the dark matter fermion mass. In addition, by combining a sub-sample of four dSphs - Draco, Fornax, Leo I and Sculptor - we conclude that $220 \mathrm{eV}$ fermionic dark matter appears to be preferred over the standard CDM at about $2 \sigma$ level. However, this result becomes insignificant if all seven objects are included in the analysis. Future improvement of the obtained bound requires more detailed data, both from 'classical' and ultra-faint dSphs.
\end{abstract}

Key words: dark matter - galaxies: haloes - galaxies: dwarf - galaxies: kinematics and dynamics - methods: statistical

\section{INTRODUCTION}

The nature of dark matter (DM) is one of the major questions in modern physics. The mass of DM particle candidates, which exist in numerous extensions of the Standard Model, varies in very wide range - from $\sim 10^{-22} \mathrm{eV}$ for ultralight DM (e.g., Hu et al. 2000; Hui et al. 2017; Lee 2018) up to TeVs for WIMPs (see, e.g., Roszkowski et al. 2018; Arcadi et al. 2018, and references therein) or up to $\sim 10^{13} \mathrm{GeV}$ for WIMPZILLAs (e.g. Chung et al. 1999).

The Pauli principle forbids packing too many fermions into a gravitationally bound object. Therefore, the average phase-space density of such an object with mass $M$ enclosed within a region of radius $R, \bar{F} \sim \frac{M}{R^{3} \sigma^{3}}$, cannot exceed some maximum $f_{\max }(m)$, where $m$ is the mass of fermion, and $\sigma$ is the particle velocity dispersion. This allows one to obtain the lower bound $m \simeq 0.5 \mathrm{keV}$ (Bode et al. 2001; Dalcanton \& Hogan 2001; Boyarsky et al. 2009; Horiuchi et al. 2014), based on the extended Tremaine-Gunn (Tremaine \& Gunn 1979) approach (see also Gorbunov et al. 2008; Shao et al. 2013; Wang et al. 2017) from the analysis of compact DM dominated objects - dwarf spheroidal satellites (dSphs).

^ E-mail: dsavchenko@bitp.kiev.ua
This approach requires an estimator of the dynamical mass $M$ within a sphere of some radius $R$ (Wolf et al. 2010; Walker \& Peñarrubia 2011; Campbell et al. 2017), see also Kowalczyk et al. (2013) for a detailed study of the mass estimator uncertainties, and Boyarsky et al. (2009) for the estimate of the phase-space volume occupied by the DM particles.

Another method for constraining the mass of the fermionic DM particle uses direct comparison between the detailed prediction of the kinematics of dSph and the observational data (see, e.g., Domcke \& Urbano 2015; Randall et al. 2017; Di Paolo et al. 2018). It does not require an estimate of the averaged phase-space density over a spatial region. Direct modelling of kinematics also allows one to incorporate the anisotropy of the velocity dispersion into analysis. Moreover, unlike the Tremaine-Gunn approach, this method allows one to combine the data on several objects to produce better limits on the particle mass. In return, it requires a (semi-)analytical model of the DM density profile and stellar density profile. Many analytical models of fermionic DM halo density profiles have been developed so far; see, e.g., Ruffini \& Stella (1983); Bilić \& Viollier (1997); Angus (2010); de Vega et al. (2014); de Vega \& Sanchez (2016); Merafina \& Alberti (2014); Domcke \& Urbano (2015); Ruffini et al. (2015); Chavanis et al. (2015); 
Argüelles et al. (2018); Randall et al. (2017); Rudakovskyi \& Savchenko (2018); Giraud \& Peschanski (2018); Barranco et al. (2018).

In this paper, we present a new lower bound on the mass of fermionic DM particle, based on the observed kinematics (Bonnivard et al. 2015) and photometry (McConnachie 2012) data of 'classical' dSphs, and assuming the DM density model of Rudakovskyi \& Savchenko (2018). In comparison to Domcke \& Urbano (2015), Di Paolo et al. (2018), this approach allows us not only to analyse individual dSphs, but also to perform combined statistical analysis based on the total $\chi^{2}$ goodness-of-fit statistics assuming the same dark matter particle mass in all of them. Thereby, we aim at utilising fully the statistical power of the approach.

This paper is organised as follows: in Sec. 2 our methods are described (a short description of our model of fermionic DM halo is also included), the obtained results are summarised in Sec. 3 and discussed in Sec. 4. We use the recent Planck (Planck Collaboration 2018) cosmological parameters for our calculations.

\section{METHODS}

We use the semi-analytical method proposed in Rudakovskyi \& Savchenko (2018) to obtain the density profile of a dark matter halo. It predicts a cored halo for the general case of warm fermionic dark matter without any extra assumptions about the particle model. Here we briefly summarise this method.

For a fermionic dark matter model with particle mass $m_{\mathrm{DM}}$ and $g$ initial degrees of freedom (hereafter, we assume $g=2$ ) the phase space density cannot exceed (Boyarsky et al. 2009)

$f_{\max }=\frac{g m_{\mathrm{DM}}^{4}}{2(2 \pi \hbar)^{3}}$

For a steady-state isotropic spherically symmetrical dark matter halo (see Rudakovskyi \& Savchenko (2018) for a discussion on the applicability of this assumption) the phase space density $f$ is obtained by using the Eddington transformation (Eddington 1916; Binney \& Tremaine 2008)

$f(\mathcal{E})=\frac{1}{\pi^{2} \sqrt{8}} \frac{d}{d \mathcal{E}} \int_{\mathcal{E}}^{0} \frac{d \rho}{d \Phi} \frac{d \Phi}{\sqrt{\mathcal{E}-\Phi}}$,

where $\Phi$ is the local gravitational potential. We perform the iterative procedure starting from the NFW profile and truncating the phase space density so that it does not exceed the limiting value:

$f_{\mathrm{tNFW}}(\mathcal{E})= \begin{cases}f(\mathcal{E}), & f(\mathcal{E})<f_{\max }, \\ f_{\max }, & f(\mathcal{E}) \geq f_{\max }\end{cases}$

After this, we reconstruct the mass density (Binney \& Tremaine 2008)

$\rho_{\mathrm{tNFW}}(r)=4 \pi \int_{\Phi(r)}^{0} f_{\mathrm{tNFW}}(\mathcal{E}) \sqrt{2(\mathcal{E}-\Phi(r))} d \mathcal{E}$

for the subsequent step. Rudakovskyi \& Savchenko (2018) shows good convergence of this procedure after several iterations. We call the obtained profile tNFW (stands for truncated Navarro-Frenk-White) hereafter. The density profiles obtained in this model are in a good agreement with numerical $N$-body simulations (Shao et al. 2013; Macciò et al. 2013a,b), see more in Rudakovskyi \& Savchenko (2018).

Given the density distribution of a dark matter halo, we follow the logic of Domcke \& Urbano (2015) and Di Paolo et al. (2018) to obtain the velocity dispersion along the line of sight. Specifically, we solve the spherical Jeans equation for the radial velocity dispersion $\sigma_{\mathrm{r}}$,

$\left(\frac{\partial}{\partial r}+\frac{2 \beta}{r}\right)\left(n_{\star} \sigma_{\mathrm{r}}^{2}\right)=-n_{\star} \frac{G M(r)}{r^{2}}$,

with the stellar velocity dispersion anisotropy $\beta=1-\sigma_{\perp}^{2} / \sigma_{\mathrm{r}}^{2}$. In the above, $M(r)$ is the dark matter mass distribution, and $n_{\star}$ is the stellar number density, which we represent by the Plummer profile (Plummer 1911)

$n_{\star}(r)=n_{0}\left(1+r^{2} / r_{\mathrm{h}}^{2}\right)^{-5 / 2}$.

The half-light radii $r_{h}$ for the objects of interest were taken from McConnachie (2012) and are given in Table 1. We then calculate the velocity dispersion along the line of sight:

$\sigma_{\mathrm{los}}^{2}(R)=\frac{1}{\Sigma_{\star}} \int_{R^{2}}^{\infty} \mathrm{d} r^{2} \frac{n_{\star}}{\sqrt{r^{2}-R^{2}}} \sigma_{\mathrm{r}}^{2}\left[1-\beta \frac{R^{2}}{r^{2}}\right]$,

where $\Sigma_{\star}(R)=\int_{R^{2}}^{\infty} \mathrm{d} r^{2} n_{\star}(r) / \sqrt{r^{2}-R^{2}}$ (Binney \& Tremaine 2008; Di Paolo et al. 2018).

We model the binned data on the velocity dispersion for eight classical dSphs taken from Bonnivard et al. (2015). For every mass of the dark matter particle in the $100 \mathrm{eV}-$ $900 \mathrm{eV}$ range with logarithmic split we use brute-force grid optimisation over the tNFW profile parameters $c_{200}, M_{200}$, and velocity dispersion anisotropy $\beta$ to minimise the objective $\chi^{2}$ statistics

$\chi^{2}=\sum_{i} \frac{\left(\sigma_{\mathrm{los}, \mathrm{obs}}\left(r_{i}\right)-\sigma_{\mathrm{los}, \mathrm{th}}\left(r_{i}\right)\right)^{2}}{\delta^{2}\left(r_{i}\right)}$,

where $\sigma_{\text {los,obs }}\left(r_{i}\right)$ denotes the $i$ 'th observational point, $\delta^{2}\left(r_{i}\right)$ is its $1 \sigma$ error, and the $\sigma_{\text {los,th }}\left(r_{i}\right)$ is the predicted value at this point; the summation is performed over the observational points.

\section{RESULTS}

The dependence of the best-fitting $\chi^{2}$ statistics on the particle mass for every individual object is plotted in Fig. 1, and the best-fitting model parameters are summarised in Table 1.

The goodness-of-fit is acceptable for every object except Carina dSph, which is the only dSph from our selection that has best-fitting $\chi^{2}$ higher than two standard deviations $\left(2 \sqrt{2 N_{\mathrm{df}}}\right)$ above the mean value $\chi_{\text {mean }}^{2}=N_{\mathrm{df}}$ of the chi-squared distribution. Therefore, we exclude Carina dSph from the subsequent combined analysis.

Apart from the individual fits, we are interested in the combined goodness-of-fit. We consider the overall $\chi^{2}$ to be the sum of chi-squared statistics of the individual fits for every dark matter particle mass. The overall best fit is obtained for the particle mass of $342 \mathrm{eV}$ with $\chi^{2}=124.7$ for 134 degrees of freedom. This value of mass, however, cannot be statistically distinguished from the higher values, as the 

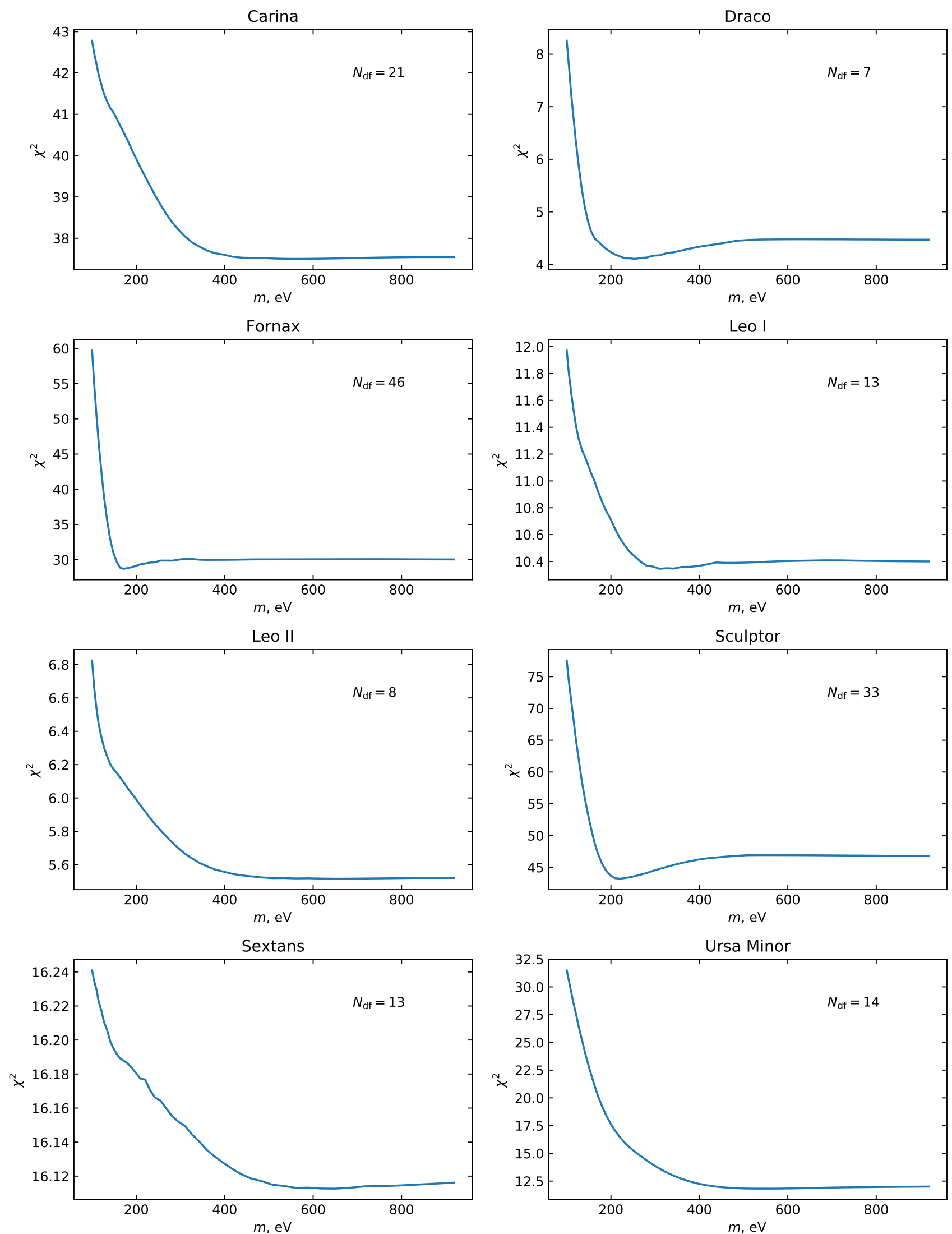

Figure 1. Minimal values of $\chi^{2}$ statistics as functions of the DM particle mass for the tNFW profile model for each of the eight 'classical' $\mathrm{d}$ Sphs studied in this paper. Also given is the number of the degrees of freedom of the fits. Notice the clearly visible minimums in four objects: Draco, Fornax, Leo I, Sculptor. 


\begin{tabular}{cccccccc} 
Object & $r_{\mathrm{h}}, \mathrm{kpc}$ & $\chi^{2} / N_{\mathrm{df}}$ & $m_{\mathrm{DM}}, \mathrm{eV}$ & $\boldsymbol{M}_{200}, 10^{8} \boldsymbol{M}_{\odot}$ & $c_{200}$ & $\beta$ & $r_{\mathrm{c}}, \mathrm{kpc}$ \\
\hline Carina & 0.25 & $37.5 / 21$ & 561 & 111.7 & 5 & 0.21 & 0.25 \\
Draco & 0.221 & $4.1 / 7$ & 255 & 177.8 & 10 & 0.34 & 0.66 \\
Fornax & 0.71 & $28.7 / 46$ & 171 & 9.57 & 53 & -0.05 & 0.93 \\
Leo1 & 0.251 & $10.4 / 13$ & 310 & 155.7 & 8 & 0.44 & 0.54 \\
Leo2 & 0.176 & $5.5 / 8$ & 650 & 127.6 & 9 & 0.61 & 0.17 \\
Sculptor & 0.283 & $43.2 / 33$ & 220 & 6.01 & 59 & 0.10 & 0.59 \\
Sextans & 0.695 & $16.1 / 13$ & 650 & 875.6 & 2 & -0.38 & 0.22 \\
Ursa Minor & 0.181 & $11.8 / 14$ & 561 & 4.92 & 36 & -1.32 & 0.15
\end{tabular}

Table 1. The best-fitting parameter values for the modelled objects and goodness-of-fit statistics. Profile parameters correspond to the Navarro-Frenk-White profile used as the starting one in the tNFW generation procedure. Also provided is the half-light radii used in our fits. Core radii $r_{c}$ for density profiles with the best-fitting parameters, are calculated from $\rho_{\mathrm{tNFW}}\left(r_{\mathrm{c}}\right)=\frac{\rho_{\mathrm{tNFW}}(0)}{4}$ according to the definition in Rudakovskyi \& Savchenko (2018).

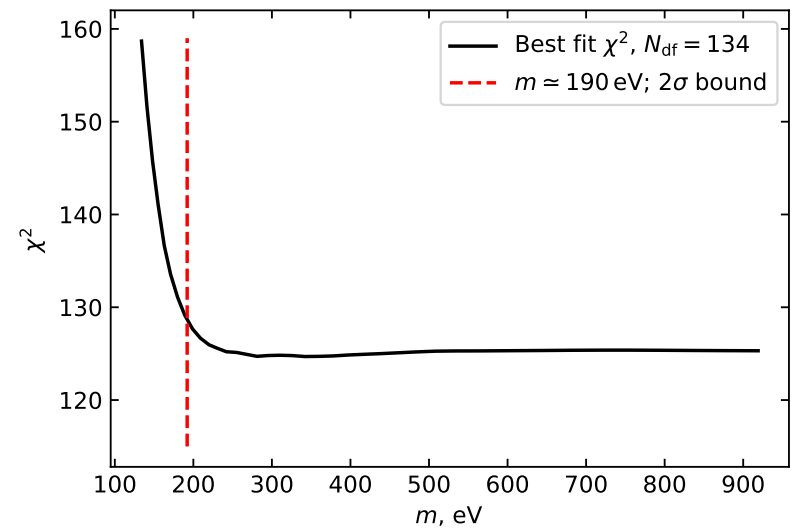

Figure 2. Overall best-fitting $\chi^{2}$ statistics as a function of the dark matter particle mass. In the limit of high mass the curve approaches the value obtained in the fit with the Navarro-FrenkWhite profile, as the tNFW halo model approaches that of the $\mathrm{NFW}$ in this limit. The dashed line shows the $2 \sigma$ confidence bound on the particle mass.

differences between the corresponding chi-squares are negligible. For comparison, we fitted the data using the NavarroFrenk-White profile (Navarro et al. 1996b, 1997), typical to the standard CDM dark matter model. The best-fitting $\chi^{2}$ statistics is 125.1 for 134 degrees of freedom, so none of this models is preferred by our analysis.

Using the dependence of the overall best-fitting statistics on the particle mass, we can build the confidence range for the mass via the standard approach, described in Sec. 15.6 of Press et al. (2007). The lower bound on the particle mass is the value for which $\chi^{2}=\chi_{\text {best-fit }}^{2}+\Delta \chi^{2}$, where for $2 \sigma$ confidence level $\Delta \chi^{2}=4$. The resulting mass bound of $m_{2 \sigma} \simeq 190 \mathrm{eV}$ is shown in Fig. 2 .

In Fig. 4 we show the effect of particle mass on the velocity dispersion profile in all objects. It is clearly seen that small particle masses strongly modify this profile.

We also combine four objects that show notable local minimum on $\chi^{2}$ vs mass dependence, namely, Draco, Fornax, Leo1 and Sculptor. The combined fitting statistics in this case is plotted in Fig. 3. The minimum $\chi_{\min }^{2}=87.4$ for 99 degrees of freedom is obtained for particle mass $m=220 \mathrm{eV}$, whereas the Navarro-Frenk-White profile fits the data with $\chi^{2}=91.4$. Thus one can conclude that $220 \mathrm{eV}$ fermionic dark

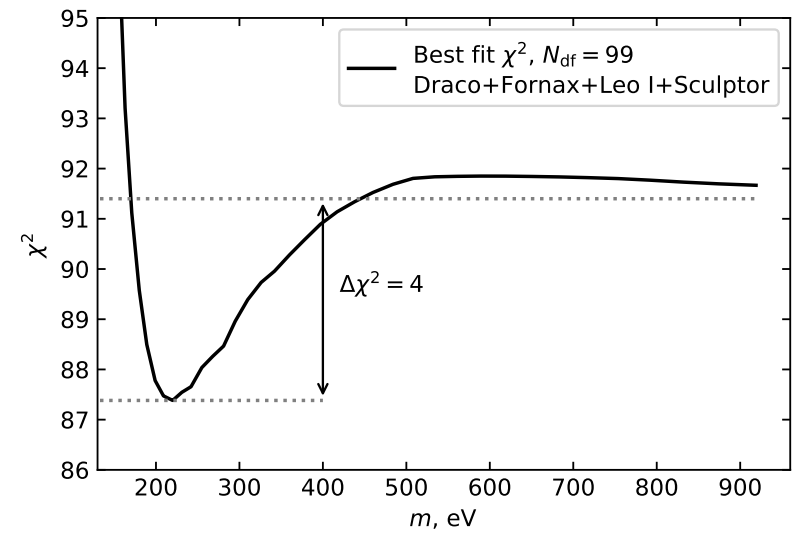

Figure 3. Overall best-fitting $\chi^{2}$ as a function of dark matter particle mass for the combined analysis of only four selected objects: Draco, Fornax, Leo1 and Sculptor. The minimum at $220 \mathrm{eV}$ indicating the preferred particle mass is clearly visible. The depth of the dip corresponds to $2 \sigma$ significance $\left(\Delta \chi^{2}=4\right)$. However, it becomes negligibly small $\left(\Delta \chi^{2}=0.4\right)$ when the rest of objects are included into analysis, see Fig. 2.

matter is preferred over CDM with $\Delta \chi^{2}=4$. However, this observation should not be treated as a strict result, because inclusion of the rest of objects into analysis reduces the local minimum to a statistically insignificant depth of $\Delta \chi^{2}=0.4$.

\section{CONCLUSIONS \& DISCUSSION}

In this paper, we derive a new maximally model-independent bound on the mass of fermionic dark matter particle. We use the halo model of Rudakovskyi \& Savchenko (2018) and the Jeans equation for modelling the line-of-sight velocity dispersion. We obtain the conservative $2 \sigma$ lower bound $m \gtrsim 190 \mathrm{eV}$ on the mass of fermionic dark matter particle. Fermionic DM with higher particle mass cannot be distinguished from the CDM. This result is based on the analysis of seven 'classical' dSphs. Our model fails to fit the kinematics of the Carina dSph. However, this galaxy shows the strongest signs of a tidal disruption among the other 'classical' dSphs (Muñoz et al. 2006, 2008; Battaglia et al. 2012, 2013; Fabrizio et al. 2016), see also McMonigal et al. (2014). It appears that Carina was transformed from a disky galaxy 

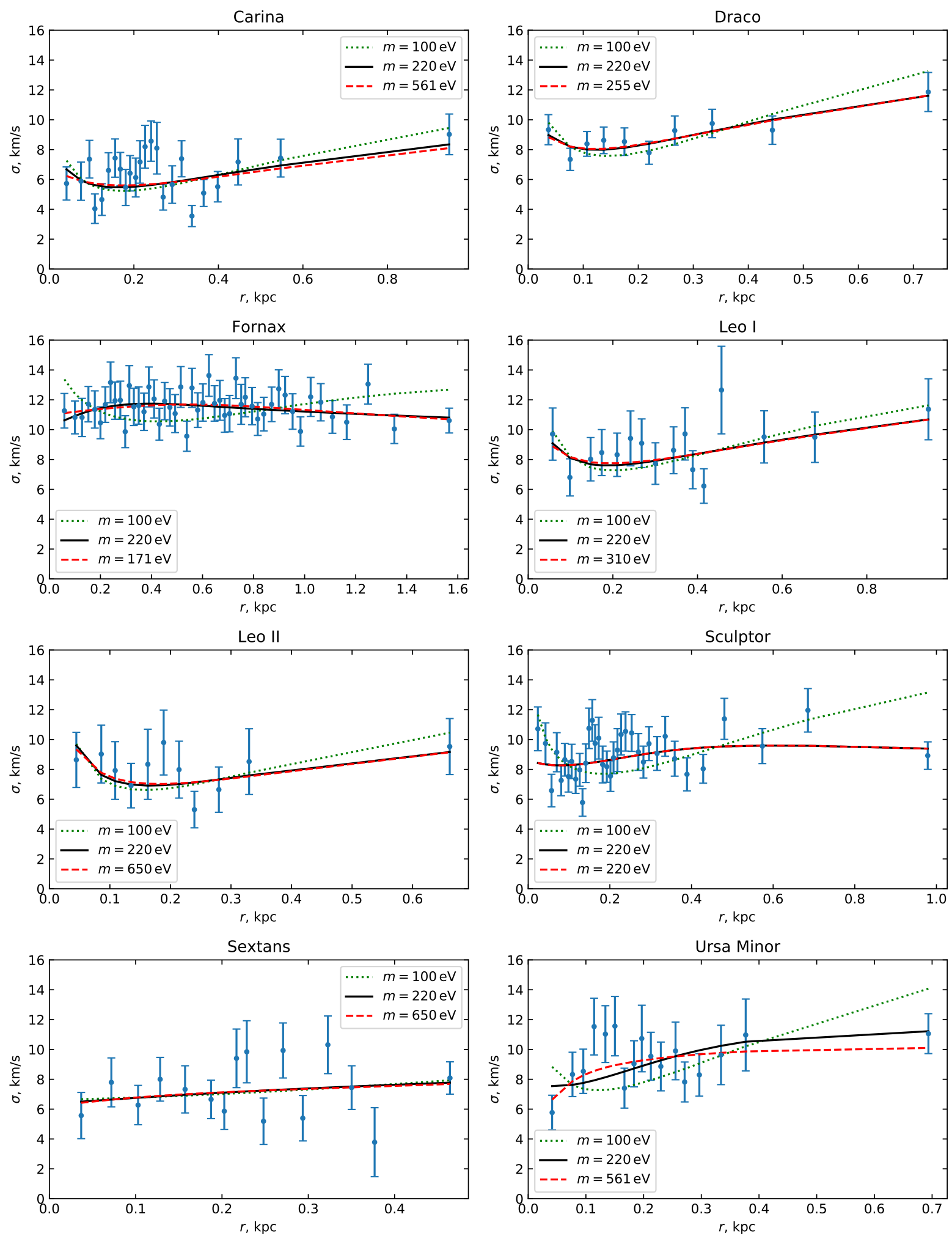

Figure 4. Velocity dispersion along the line of sight versus the distance from the object centre. The dots with error bars represent the binned observational data, taken from Bonnivard et al. (2015). The lines show the best-fitting dependence obtained in the tNFW model with different particle masses, namely, $m=100 \mathrm{eV}$ which is below the obtained $2 \sigma$ bound, $m=220 \mathrm{eV}$ preferred by the combined analysis of four selected dSphs (Draco, Fornax, Leo I, Sculptor), and the mass which provides the minimal $\chi 2$ in the fit of the corresponding individual object. One can see that for most of objects the behaviour of $\sigma_{\text {los }}$ in the case of low particle mass strongly changes whereas variation of mass above the $190 \mathrm{eV}$ bound has small impact on this behaviour. 
to a spheroidal via strong tidal interaction with Milky Way (Fabrizio et al. 2016), and different sub-populations have different kinematic patterns (Fabrizio et al. 2016; Hayashi et al. 2018).

To check the robustness of the obtained result with respect to possible uncertainties in the values of half-light radii, we repeat the analysis using upper and lower confidence bounds on $r_{\mathrm{h}}$ reported by McConnachie (2012). We found that the obtained lower bound on the particle mass changes by less than $10 \%$, being lower for the higher $r_{h}$ used in the model and vice-versa.

Using only the data on four selected objects, namely, Draco, Fornax, Leo1 and Sculptor, we obtain that fermionic DM with $m=220 \mathrm{eV}$ particle mass is preferred over CDM on $2 \sigma$ level. The significance decreases to a negligible value when the rest of objects are included into the analysis.

Conceptually, the halo built from the low-mass fermions has an extended core with low central density compared to the cases of more massive DM particles. The best-fitting halos in case of fermions with the mass $m=100 \mathrm{eV}$ show $\sim 2 \mathrm{kpc}$ cores for all the objects in the analysis. This is much larger than the radial spans of the outermost points of the observable kinematics.

The behaviour of $\sigma_{\text {los }}$ is determined by the behaviour of $\sigma_{\mathrm{r}}$, which is smoothed on the characteristic scale $r_{h}$ via integral transformation according to Eq. 7, see Fig. 6, 7. Therefore, in the following discussion we will focus on the behaviour of the radial velocity dispersion.

Eq. 5 could be rewritten in the form analogous to Eq. 14 of Di Paolo et al. (2018):

$\frac{\partial \ln \sigma_{\mathrm{r}}^{2}}{\partial \ln r}=-\frac{1}{\sigma_{\mathrm{r}}^{2}} \frac{G M(r)}{r}-\frac{\partial \ln n_{\star}}{\partial \ln r}-2 \beta$.

According to this equation, the logarithmic slope of $\sigma_{\mathrm{r}}$ depends on three different terms. The first negative term dominates on large scales. On the scales $\gtrsim r_{\mathrm{h}}$, the influence of the second positive term $-\frac{\partial \ln n_{\star}}{\partial \ln r}=5 r^{2} /\left(r^{2}+r_{h}^{2}\right)$ is also significant. A density profile with a few-kpc core is similar to a constant-density profile for $r \ll r_{\mathrm{c}}$, and such halo has much lower mass enclosed into radii $\lesssim 1 \mathrm{kpc}$ compared to a more cusped one (see Fig. 6, 7). In this case the first term in Eq. 9 is larger on the scales $\lesssim 1 \mathrm{kpc}$ compared to the case of more dense halos. Therefore, the logarithmic slope of $\sigma_{\mathrm{r}}$ is larger for halos built from low-mass fermions on the scales $r_{\mathrm{h}} \lesssim r \lesssim r_{\mathrm{c}}$. Such slope is not compatible with the data, and could be partially corrected by the third term of the Eq. 9 with positive $\beta$. However, large positive $\beta$ leads to fast decreasing profile of $\sigma_{\mathrm{r}}$ for such halos in the low- $r$ region ${ }^{1}$. The dip produced in this case is reflected in the profile of $\sigma_{\text {los }}$, which also limits the ability to choose very large $\beta$. Generally speaking, the discussed behaviour of the radial and line-ofsight velocity dispersions is reflected in the decrease of the best-fitting $\beta$ values with an increase of $r_{\mathrm{c}} / r_{\mathrm{h}}$, see Table 2 .

Despite the large spread of the neighbouring points, the observational data can be regarded as "flat", i.e. preferring $\sigma_{\text {los }}$ profiles without large dips or high slope. Taking into ac-

1 The asymptotic behaviour of the radial velocity dispersion is $\sigma_{\mathrm{r}} \sim r^{-2 \beta}+C r^{2}$ in the region of small $r$ for cored halos, where $C$ is some constant. count the discussion above, one can conclude that the halos built with low-mass particles contradict such "flatness".

In this context we must mention that Sextans has the largest scatter between the nearby observational points among other dSphs. The values in the neighbouring points often have more than 1-sigma differences. Also, the value of $r_{\mathrm{h}}$ in Sextans is about $0.7 \mathrm{kpc}$, or 1.5 times larger than the maximal radial span $\left(r_{\max }\right)$ of the available kinematic data. As we mentioned above, $\sigma_{\text {los }}$ is smoothed against $\sigma_{\mathrm{r}}$ with the characteristic radius $r_{\mathrm{h}}$. Because the ratio of $r_{\max } / r_{\mathrm{h}}$ for Sextans is the smallest among the classical dSphs, the corresponding level of sigma $a_{\text {los }}$ "smoothness" is the largest. This leads to the fact that all best-fits have close values of goodness-of-fit statistics and similar shape. In contrast, objects with sufficiently large $r_{\max } / r_{\mathrm{h}}$ ratios (such as Sculptor, Ursa Minor, Fornax) demonstrate the largest variations among profiles with different values of the dark matter masses.

Also note that our best-fitting parameters for Fornax $m=171 \mathrm{eV}, M_{200}=9.57 \cdot 10^{8} M_{\odot}, C_{200}=53$ correspond to a profile with $r_{\mathrm{C}}=0.93 \mathrm{kpc}$, which is in a good agreement with Amorisco et al. $(2013)^{2}$. While, in general, our analysis shows no significant preference for the cored dark matter profiles over the cusped ones (obtained in the $\Lambda C D M$ model), Draco, Fornax, Leo1 and Sculptor may give a possible hint on such preference.

The main advantage of our analysis is the combined study of several objects: we simultaneously fit the data for seven classical dSphs. While the fits of the data of individual objects show different preferred particle masses (see Fig. 1) and lead to different bounds, the combined analysis ensures robustness of the results. Moreover, when modelling several object, we are able to produce stronger bound. For example, the strongest limit of $100 \mathrm{eV}$ in Di Paolo et al. (2018) is obtained by analysing the smallest dwarfs, whereas the analysis of the classical dwarfs only leads to the mass limit of few tens of electron-volts.

In general, the dark matter halo profile of Di Paolo et al. (2018) systematically prefers lower particle masses due to its fully degenerate nature, which produces sharp cut-off in the density profile. Unlike in Di Paolo et al. (2018), in our model the DM halo has two regions: a fully degenerate core and non-degenerate dispersed outskirts. Fig. 5 shows the fast clipping of this profile and the smaller core size, compared with more "blured" tNFW profile.

Recent direct measurements of 3D stellar kinematics in Sculptor (Massari et al. 2018) and kinematics data modelling via the Schwarzschild method (Kowalczyk et al. 2019) revealed that the stellar velocity dispersions in the dwarf spheroidal galaxies are likely to be non-isotropic, but the uncertainties in the value of $\beta$ are very large. Therefore, we assume, for simplicity, that this quantity is constant on all radii. Inclusion of non-zero stellar velocity anisotropy into the analysis leads to a lower DM mass bound compared to the previous findings (e.g. Boyarsky et al. 2009). In the case of non-zero $\beta$, we found that DM particle masses in wide range are statistically indistinguishable. This agrees quali-

2 Despite that Amorisco et al. (2013) used the Burkert profile, the core radius $r_{0}$ is defined as $\rho\left(r_{0}\right)=\frac{\rho_{0}}{4}$, which is similar to our definition 


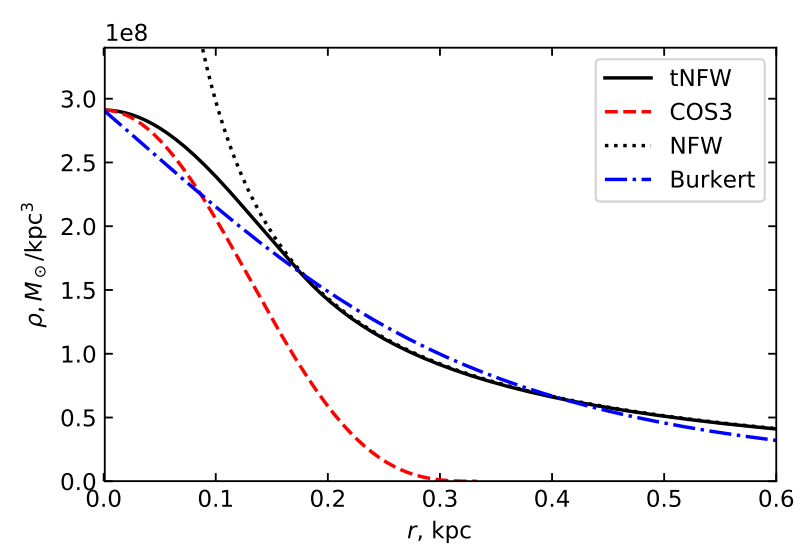

Figure 5. Dark matter density profile in the tNFW model with particle mass $m=380 \mathrm{eV}, M_{200}=1.5 \times 10^{10} M_{\odot}, c_{200}=10$ compared with the fully degenerate COS3 profile of Di Paolo et al. (2018) with the same central density and particle mass. The NFW profile here has the corresponding asymptotic behaviour. Also plotted is the Burkert cored profile Burkert (1995) with $r_{0}=0.38 \mathrm{kpc}$ (twice the fully degenerate COS3 profile core size).

tatively with the results of Di Paolo et al. (2018) and Randall et al. (2017) for models of non-fully degenerate fermionic halos. This $\beta$-degeneracy could be overcome by assuming multiple stellar sub-populations (Battaglia et al. 2008; Walker \& Peñarrubia 2011; Agnello \& Evans 2012; Amorisco et al. 2013) or by using the Virial equations instead of the Jeans equations (Richardson \& Fairbairn 2014). However, the existing data, which does not include proper 3D stellar kinematics with possible asphericity of stellar populations, is not enough to completely break this degeneracy (Kowalczyk et al. 2013; Genina et al. 2018; Hayashi et al. 2018).

The effects of supernova feedback (Navarro et al. 1996a; Pontzen \& Governato 2012; Oh et al. 2011; Teyssier et al. 2013; Zolotov et al. 2012), other stellar feedback mechanisms (Chan et al. 2015; Oñorbe et al. 2015), and dynamical friction (El-Zant et al. 2004; Sánchez-Salcedo et al. 2006; Romano-Díaz et al. 2008; Del Popolo \& Pace 2016) could cause additional flattening of the dark matter profile and reduction of the central phase-space density. These mechanisms are thus degenerate with the dark-matter-induced core generation. Inclusion of these effects could increase the lower mass bound.

In the future, progress in the exploration of DM microphysics may be achieved via studying the ultra-faint dwarfs (UFDs), which are the most DM dominated galaxies that we know (see, e.g., Bullock \& Boylan-Kolchin 2017; Simon 2019). Their compactness also gives an opportunity to test the dark matter distribution on the smallest scales, e.g., dozens of parsecs. Also, the star-formation processes in UFDs should not be powerful enough to change substantially their internal density structure (Oñorbe et al. 2015). However, even the most recent studies (e.g., Fritz et al. 2018; Simon 2018) allow one to obtain spectra only for only dozens of stars in the ultra-faint Milky Way satellites (unlike 'classical' dwarfs, where spectra of hundreds or thousands of stars are measured). These data are not enough to obtain any detailed line-of-sight velocity dispersion profile. Lengthy ob-

\begin{tabular}{lcccccc} 
Object & $\mathrm{m}, \mathrm{eV}$ & $m_{200}$ & $c_{200}$ & $\beta$ & $r_{\mathrm{c}}, \mathrm{kpc}$ & $r_{\mathrm{h}}, \mathrm{kpc}$ \\
\hline Carina & 100 & 35.98 & 120 & 0.74 & 2.11 & 0.25 \\
Carina & 220 & 119.4 & 6 & 0.52 & 0.99 & 0.25 \\
Carina & 650 & 111.7 & 5 & 0.19 & 0.20 & 0.25 \\
& & & & & & \\
Draco & 100 & 69.47 & 120 & 0.67 & 1.80 & 0.221 \\
Draco & 220 & 119.4 & 12 & 0.40 & 0.78 & 0.221 \\
Draco & 650 & 717.4 & 6 & 0.10 & 0.17 & 0.221 \\
& & & & & & \\
Fornax & 100 & 33.92 & 200 & 0.41 & 2.10 & 0.710 \\
Fornax & 220 & 10.93 & 26 & -0.24 & 0.67 & 0.710 \\
Fornax & 650 & 13.34 & 19 & -0.50 & 0.15 & 0.710 \\
& & & & & & \\
Leo1 & 100 & 52.27 & 200 & 0.83 & 1.87 & 0.251 \\
Leo1 & 220 & 145.7 & 9 & 0.58 & 0.86 & 0.251 \\
Leo1 & 650 & 127.6 & 8 & 0.27 & 0.18 & 0.251 \\
& & & & & & \\
Leo2 & 100 & 76.32 & 85 & 1.00 & 1.82 & 0.176 \\
Leo2 & 220 & 111.7 & 12 & 0.85 & 0.78 & 0.176 \\
Leo2 & 650 & 127.6 & 9 & 0.61 & 0.17 & 0.176 \\
& & & & & & \\
Sculptor & 100 & 52.27 & 200 & 0.64 & 1.87 & 0.283 \\
Sculptor & 220 & 6.010 & 59 & 0.10 & 0.59 & 0.283 \\
Sculptor & 650 & 11.68 & 19 & -0.38 & 0.15 & 0.283 \\
& & & & & & \\
Sextans & 100 & 25.91 & 77 & -0.05 & 2.34 & 0.695 \\
Sextans & 220 & 145.7 & 4 & -0.20 & 1.11 & 0.695 \\
Sextans & 650 & 875.6 & 2 & -0.38 & 0.22 & 0.695 \\
& & & & & & \\
UMi & 100 & 80.55 & 160 & 0.69 & 1.70 & 0.181 \\
UMi & 220 & 6.012 & 160 & 0.06 & 0.52 & 0.181 \\
UMi & 650 & 4.311 & 38 & -1.64 & 0.12 & 0.181 \\
& & & & & &
\end{tabular}

Table 2. Best-fitting values of the halo profile parameters and halo core size $r_{\mathrm{c}}$ for three fixed dark matter particle masses: $100 \mathrm{eV}, 220 \mathrm{eV}$, and $650 \mathrm{eV}$. Half-light radii $r_{h}$ are also given for reference. The $m_{200}$ values are in $10^{8} M_{\odot}$.

servations on $\sim 10 \mathrm{~m}$ or planned extremely large telescopes may obtain the spectra of many more stars (Strigari 2018; Weisz \& Boylan-Kolchin 2019; Drlica-Wagner et al. 2019; Simon et al. 2019).

\section{ACKNOWLEDGEMENTS}

The authors are grateful to D. Iakubovskyi and Yu. Shtanov for collaboration and valuable comments. We thank the anonymous Referee for the comments that significantly improved the quality of the paper. This work was supported by the grant for young scientist's research laboratories of the National Academy of Sciences of Ukraine. The work of A.R. was also partially supported by the ICTP through AF-06.

\section{REFERENCES}

Agnello A., Evans N. W., 2012, ApJ, 754, L39

Amorisco N. C., Agnello A., Evans N. W., 2013, MNRAS, 429, L89

Angus G. W., 2010, J. Cosmology Astropart. Phys., 3, 026

Arcadi G., Dutra M., Ghosh P., Lindner M., Mambrini Y., Pierre M., Profumo S., Queiroz F. S., 2018, Eur. Phys. J., C78, 203 Argüelles C. R., Krut A., Rueda J. A., Ruffini R., 2018, Phys. Dark Univ., 21, 82 

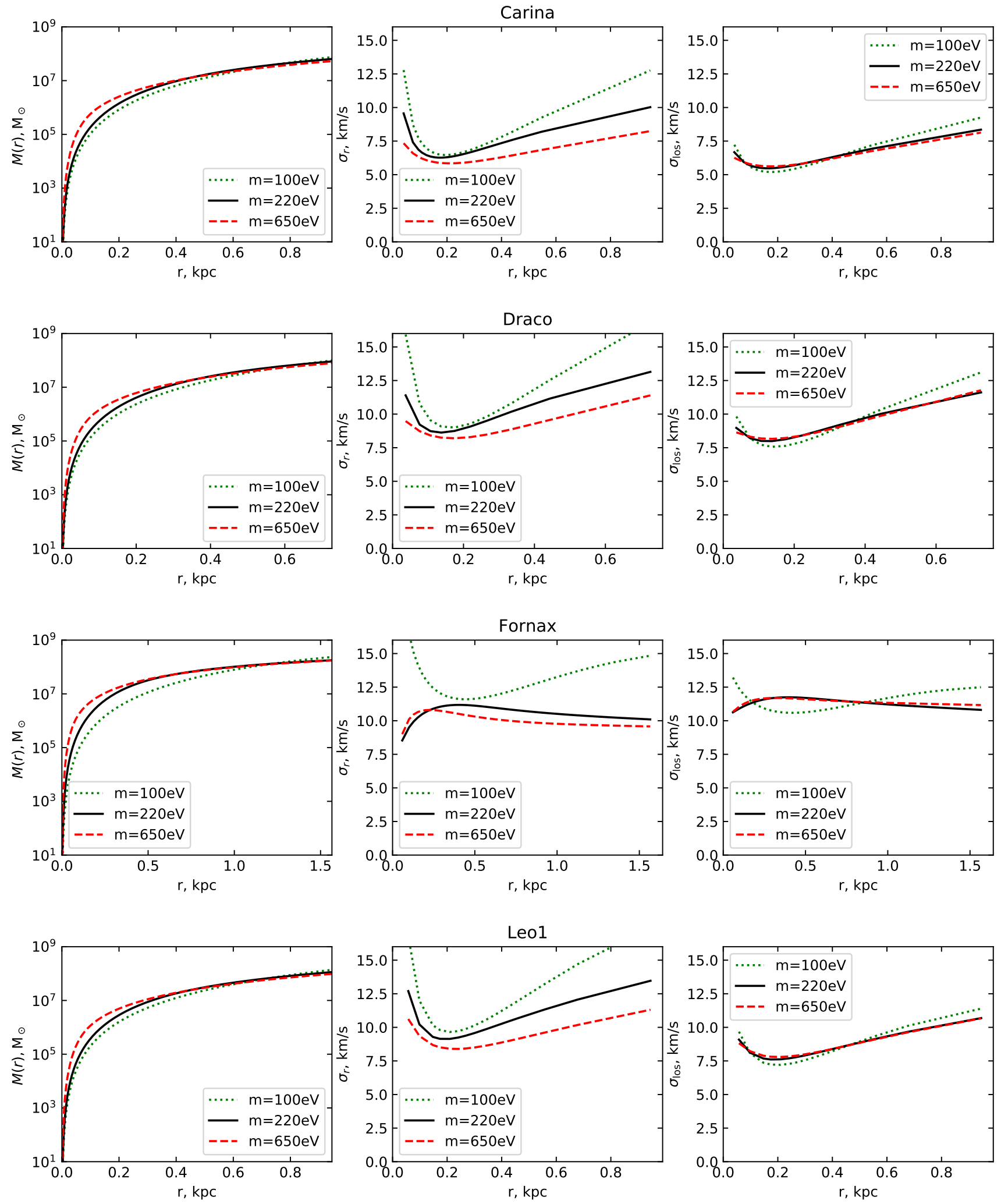

Figure 6. The dependence of the enclosed mass, radial velocity anisotropy, line-of-sight velocity anisotropy from the off-center distance for the three fixed particle masses, same as in Table 2. First four dSphs from the analysis shown. 

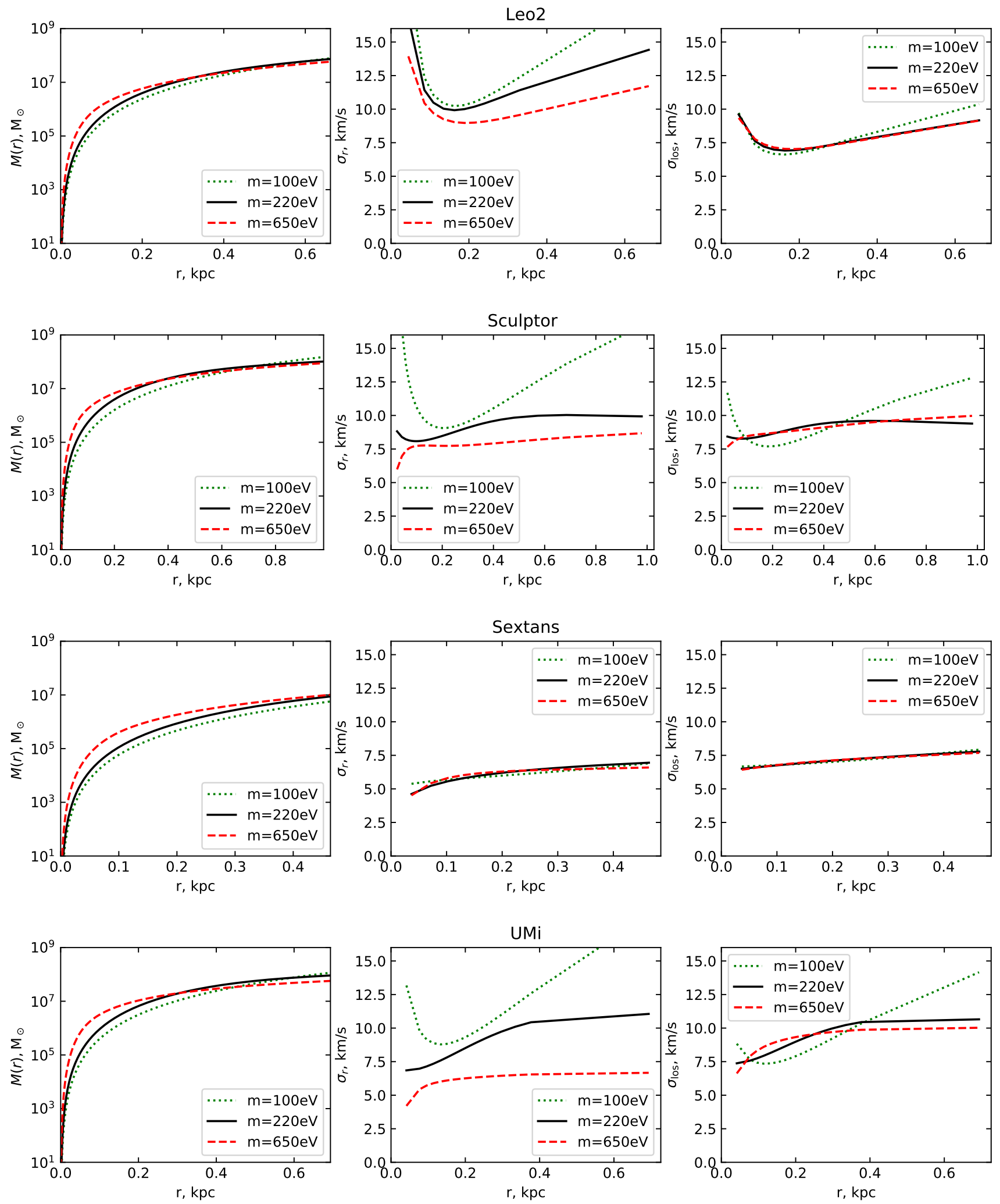

Figure 7. Same as in Fig. 6 but for another four objects. 
Barranco J., Bernal A., Delepine D., 2018, preprint (arXiv:1811.11125)

Battaglia G., Helmi A., Tolstoy E., Irwin M., Hill V., Jablonka P., 2008, ApJ, 681, L13

Battaglia G., Irwin M., Tolstoy E., de Boer T., Mateo M., 2012, ApJ, 761, L31

Battaglia G., Helmi A., Breddels M., 2013, New Astronomy Reviews, 57,52

Bilić N., Viollier R. D., 1997, Phys. Lett. B, 408, 75

Binney J., Tremaine S., 2008, Galactic Dynamics: Second Edition. Princeton University Press, Princeton, NJ USA

Bode P., Ostriker J. P., Turok N., 2001, ApJ, 556, 93

Bonnivard V., et al., 2015, MNRAS, 453, 849

Boyarsky A., Ruchayskiy O., Iakubovskyi D., 2009, J. Cosmology Astropart. Phys., 3, 005

Bullock J. S., Boylan-Kolchin M., 2017, ARA\&A, 55, 343

Burkert A., 1995, ApJ, 447, L25

Campbell D. J. R., et al., 2017, MNRAS, 469, 2335

Chan T. K., Kereš D., Oñorbe J., Hopkins P. F., Muratov A. L., Faucher-Giguère C.-A., Quataert E., 2015, MNRAS, 454, 2981

Chavanis P.-H., Lemou M., Méhats F., 2015, Phys. Rev. D, 92, 123527

Chung D. J. H., Kolb E. W., Riotto A., 1999, Phys. Rev. D, 59, 023501

Dalcanton J. J., Hogan C. J., 2001, ApJ, 561, 35

Del Popolo A., Pace F., 2016, Ap\&SS, 361, 162

Di Paolo C., Nesti F., Villante F. L., 2018, MNRAS, 475, 5385

Domcke V., Urbano A., 2015, J. Cosmology Astropart. Phys., 1501, 002

Drlica-Wagner A., et al., 2019, preprint (arXiv: 1902.01055)

Eddington A. S., 1916, MNRAS, 76, 572

El-Zant A. A., Hoffman Y., Primack J., Combes F., Shlosman I., 2004, ApJ, 607, L75

Fabrizio M., et al., 2016, ApJ, 830, 126

Fritz T. K., Battaglia G., Pawlowski M. S., Kallivayalil N., van der Marel R., Sohn S. T., Brook C., Besla G., 2018, A\&A, 619, A103

Genina A., et al., 2018, MNRAS, 474, 1398

Giraud B. G., Peschanski R., 2018, preprint (arXiv: 1806.07283)

Gorbunov D., Khmelnitsky A., Rubakov V., 2008, J. Cosmology Astropart. Phys., 2008, 041

Hayashi K., Fabrizio M., Łokas E. L., Bono G., Monelli M., Dall'Ora M., Stetson P. B., 2018, MNRAS, 481, 250

Horiuchi S., Humphrey P. J., Oñorbe J., Abazajian K. N., Kaplinghat M., Garrison-Kimmel S., 2014, Phys. Rev. D, 89, 025017

Hu W., Barkana R., Gruzinov A., 2000, Phys. Rev. Lett., 85, 1158

Hui L., Ostriker J. P., Tremaine S., Witten E., 2017, Phys. Rev. D, 95, 043541

Kowalczyk K., Łokas E. L., Kazantzidis S., Mayer L., 2013, MNRAS, 431, 2796

Kowalczyk K., del Pino A., Łokas E. L., Valluri M., 2019, MNRAS, 482,5241

Lee J.-W., 2018, in European Physical Journal Web of Conferences. p. 06005 (arXiv: 1704.05057), doi:10.1051/epjconf/201816806005

Macciò A. V., Ruchayskiy O., Boyarsky A., Muñoz-Cuartas J. C., 2013a, MNRAS, 428, 882

Macciò A. V., Paduroiu S., Anderhalden D., Schneider A., Moore B., 2013b, MNRAS, 428, 3715

Massari D., Breddels M. A., Helmi A., Posti L., Brown A. G. A., Tolstoy E., 2018, Nature Astronomy, 2, 156

McConnachie A. W., 2012, AJ, 144, 4

McMonigal B., et al., 2014, MNRAS, 444, 3139

Merafina M., Alberti G., 2014, Phys. Rev. D, 89, 123010

Muñoz R. R., et al., 2006, ApJ, 649, 201

Muñoz R. R., Majewski S. R., Johnston K. V., 2008, ApJ, 679, 346
Navarro J. F., Eke V. R., Frenk C. S., 1996a, MNRAS, 283, L72 Navarro J. F., Frenk C. S., White S. D. M., 1996b, ApJ, 462, 563 Navarro J. F., Frenk C. S., White S. D. M., 1997, ApJ, 490, 493

Oñorbe J., Boylan-Kolchin M., Bullock J. S., Hopkins P. F., Kereš D., Faucher-Giguère C.-A., Quataert E., Murray N., 2015, MNRAS, 454, 2092

Oh S.-H., Brook C., Governato F., Brinks E., Mayer L., de Blok W. J. G., Brooks A., Walter F., 2011, AJ, 142, 24

Planck Collaboration 2018, preprint (arXiv:1807.06209)

Plummer H. C., 1911, MNRAS, 71, 460

Pontzen A., Governato F., 2012, MNRAS, 421, 3464

Press W. H., Teukolsky S. A., Vetterling W. T., Flannery B. P., 2007, Numerical Recipes 3rd Edition: The Art of Scientific Computing, 3 edn. Cambridge University Press, New York, NY, USA

Randall L., Scholtz J., Unwin J., 2017, MNRAS, 467, 1515

Richardson T., Fairbairn M., 2014, MNRAS, 441, 1584

Romano-Díaz E., Shlosman I., Hoffman Y., Heller C., 2008, ApJ, 685, L105

Roszkowski L., Sessolo E. M., Trojanowski S., 2018, Rept. Prog. Phys., 81, 066201

Rudakovskyi A., Savchenko D., 2018, Ukr. J. Phys., 63, 769

Ruffini R., Stella L., 1983, A\&A, 119, 35

Ruffini R., Argüelles C. R., Rueda J. A., 2015, MNRAS, 451, 622

Sánchez-Salcedo F. J., Reyes-Iturbide J., Hernandez X., 2006, MNRAS, 370, 1829

Shao S., Gao L., Theuns T., Frenk C. S., 2013, MNRAS, 430, 2346

Simon J. D., 2018, ApJ, 863, 89

Simon J. D., 2019, preprint (arXiv:1901.05465)

Simon J. D., et al., 2019, preprint, (arXiv:1903.04743)

Strigari L. E., 2018, Reports on Progress in Physics, 81, 056901

Teyssier R., Pontzen A., Dubois Y., Read J. I., 2013, MNRAS, 429, 3068

Tremaine S., Gunn J. E., 1979, Phys. Rev. Lett., 42, 407

Walker M. G., Peñarrubia J., 2011, ApJ, 742, 20

Wang M.-Y., Cherry J. F., Horiuchi S., Strigari L. E., 2017, preprint, (arXiv: 1712.04597)

Weisz D. R., Boylan-Kolchin M., 2019, preprint (arXiv: 1901.07571)

Wolf J., Martinez G. D., Bullock J. S., Kaplinghat M., Geha M., Muñoz R. R., Simon J. D., Avedo F. F., 2010, MNRAS, 406, 1220

Zolotov A., et al., 2012, ApJ, 761, 71

de Vega H. J., Sanchez N. G., 2016, Int. J. Mod. Phys. A, 31, 1650073

de Vega H. J., Salucci P., Sanchez N. G., 2014, MNRAS, 442, 2717

This paper has been typeset from a $\mathrm{T}_{\mathrm{E}} \mathrm{X} / \mathrm{LAT}_{\mathrm{E}} \mathrm{X}$ file prepared by the author. 